Research Paper

\title{
PLX4032 Mediated Melanoma Associated Antigen Potentiation in Patient Derived Primary Melanoma Cells
}

\author{
Andrea L. George ${ }^{1}$, Robert Suriano ${ }^{2}$, Shilpi Rajoria ${ }^{1}$, Maria C. Osso ${ }^{4}$, Neha Tuli1 ${ }^{1}$ Elyse Hanly ${ }^{1}$, Jan \\ Geliebter ${ }^{1}$, Angelo N. Arnold5 , Marc Wallack1,3, Raj K. Tiwari ${ }^{1}$ \\ 1. New York Medical College, Department of Microbiology and Immunology, Valhalla, New York, 10595; \\ 2. College of Mount Saint Vincent, Division of Natural Sciences, Bronx, New York, 10471; \\ 3. Metropolitan Hospital Center GNS, Department of Surgery, New York, New York, 10029; \\ 4. McDaniel College, Westminster, Maryland 21157; \\ 5. Westchester Medical Center, Transplant Immunogenetics Laboratory, Valhalla, New York, 10595. \\ $\triangle$ Corresponding author: Dr. Raj K. Tiwari, New York Medical College, BSB 331, 95 Grasslands Rd, Valhalla, NY 10595 Tel: (914) 594-4186, Fax: (914) \\ 594-4176 E-mail: raj_tiwari@nymc.edu.
}

(C) 2015 Ivyspring International Publisher. Reproduction is permitted for personal, noncommercial use, provided that the article is in whole, unmodified, and properly cited. See http:/ /ivyspring.com/terms for terms and conditions.

Received: 2014.11.20; Accepted: 2015.06.19; Published: 2015.10.29

\begin{abstract}
Over expression of various immunogenic melanoma associated antigens (MAAs) has been exploited in the development of immunotherapeutic melanoma vaccines. Expression of MAAs such as MART-1 and gP100 is modulated by the MAPK signaling pathway, which is often deregulated in melanoma. The protein BRAF, a member of the MAPK pathway, is mutated in over $60 \%$ of melanomas providing an opportunity for the identification and approval by the FDA of a small molecule MAPK signaling inhibitor PLX4032 that functions to inactivate mutant BRAF ${ }^{\mathrm{V} 600 \mathrm{E}}$.

To this end, we characterized five patient derived primary melanoma cell lines with respect to treatment with PLX4032. Cells were treated with $5 \mu$ M PLX4032 and harvested. Western blotting analysis, RT-PCR and in vitro transwell migration and invasion assays were utilized to determine treatment effects. PLX4032 treatment modulated phosphorylation of signaling proteins belonging to the MAPK pathway including BRAF, MEK, and ERK and abrogated cell phenotypic characteristics such as migration and invasion. Most significantly, PLX4032 led to an up regulation of many MAA proteins in three of the four BRAF mutated cell lines, as determined at the protein and RNA level. Interestingly, MAGE-Al protein and mRNA levels were reduced upon PLX4032 treatment in two of the primary lines.

Taken together, our findings suggest that the BRAF ${ }^{\mathrm{V} 600 \mathrm{E}}$ inhibitor PLX4032 has therapeutic potential over and above its known target and in combination with specific melanoma targeting vaccine strategies may have further clinical utility.
\end{abstract}

Key words: BRAF Kinases, MAPK, Melanoma, Melanoma Associated Antigens, PLX4032, Vaccine.

\section{Introduction}

According to the Center for Disease Control, skin cancer is the most common form of cancer in the U.S. of which melanoma remains the most aggressive form. More than 60,000 new cases of melanoma are diagnosed in the U.S. each year resulting in nearly 9,000 melanoma related deaths. While highly aggressive and responsible for most skin cancer related deaths, melanoma is considered one of the most immunogenic cancers, supported by various documented cases of spontaneous tumor regressions [1,2].
Furthermore, the identification and characterization of melanoma associated antigens (MAAs) as targets of immunotherapy are also well documented [3].

The various MAAs identified function in physiological processes including melanoma growth and progression and classically have been grouped into two main categories based on their biological activity. The first group consists of MAAs associated with melanosomal maturation and melanin production namely tyrosinase, TRP-1 (gp75), gp100 and MART-1. 
The second group consists of antigens collectively known as the cancer testis antigens and includes the proteins MAGE-A1 and NY-ESO-1 [4]. Importantly, tyrosinase, TRP-1 (gp75), gp100, MART-1, NY-ESO-1 and MAGE-A1 are over-expressed in melanomas, making them lucrative targets for developing immunotherapeutic approaches, including vaccines. We previously conducted an FDA and NCI approved double blinded randomized cancer vaccine clinical trial using a vaccinia virus based melanoma cell membrane preparation, containing various MAAs, which demonstrated efficacy in a subset of patients.

Previously, a phase III trial using a gp100: 209-217(210M) peptide vaccine in combination with interleukin-2 (IL-2) treatment observed an increase in overall clinical response and longer progression-free survival in melanoma patients receiving the peptide vaccine versus those receiving IL-2 alone [5]. Other strategies in melanoma vaccine development have included the use of adoptive transfer of autologous dendritic cells that have been pulsed with individual MAA's and subsequently injected into patients [6]. While MAA specific cytotoxic T cells can be isolated following these immunotherapeutic strategies, robust, long-term anti-tumor responses have not been achieved. In addition, we and others have demonstrated that not all melanomas express the various MAAs, and together with the fact that selective loss of antigens may take place, suggest that development of an effective immunotherapeutic vaccine will require a multi-valent approach to elicit and maintain a prolonged immune response and minimize immune evasion. Expression of many MAAs is regulated transcriptionally by microphthalmia-associated transcription factor (MITF), which has been shown to not only regulate melanosome maturation through control of MAA expression but also to control genes important for cell-cycle progression [7]. Further, MITF action itself is regulated by posttranslational modification via signaling of the mitogen activated protein kinase (MAPK) pathway [8].

Deregulation of the MAPK pathway is associated with melanoma development and leads to loss of control of cell cycle progression and proliferation [9]. Further, aberrant MAPK signaling in melanoma also leads to activation of STAT3 and production of immunosuppressive and angiogenic cytokines including IL-10, VEGF, and IL-6, thus modulating anti-tumor immune responses. Inhibition of BRAF or MEK reverses this suppressive effect [10]. The MAPK pathway includes kinases such as BRAF, MEK, and ERK, which in tandem phosphorylate the next protein in the cascade resulting in activation of cellular programs controlling proliferation, differentiation, migration, and apoptosis. Of the many mutations known to affect the MAPK pathway, greater than $60 \%$ of melanomas harbor an activating mutation of the BRAF protein kinase in which a thymine is substituted with adenine at nucleotide 1799 leading to an amino acid substitution of valine $(\mathrm{V})$ to glutamate $(\mathrm{E})$ at codon 600 [11]. This mutated form of BRAF $\left(\mathrm{BRAF}^{\mathrm{V} 600 \mathrm{E}}\right)$ results in constitutive activation of the mitogen-activated protein kinase (MAPK) pathway and has led to identification of small molecule inhibitors that target MAPK components.

One such small molecule, PLX4032 also known as vemurafenib (Zelboraf $\AA$, Genentech) gained FDA approval in 2011 for the treatment of patients with late stage or unresectable melanoma after promising results in clinical trials demonstrated increased progression free and overall survival when compared to patients receiving standard care of dacarbazine [12]. PLX4032 specifically targets BRAFV600E, preventing phosphorylation of MEK and subsequent signaling through the MAPK pathway. Since then, numerous other trials have also reported beneficial effects of vemurafenib. In conjunction with its deleterious effects on melanoma progression, aberrant MAPK signaling has also been linked to modulation of melanoma associated antigen expression. Boni et al. demonstrated that treatment of BRAFV600E positive melanoma cells with either a BRAF inhibitor or two specific MEK inhibitors (U0126 and PD0325901) led to increased transcription and expression of the melanoma differentiation antigen MART-1, thus indicative that constitutive MAPK signaling is able to down regulate important proteins involved in cancer cell immune detection [13]. Interestingly, this observation by which MAPK signaling alters expression of MAAs may provide a synergistic treatment strategy combining BRAF inhibitors with a multi-valent immunotherapeutic melanoma vaccine. To that end, we have focused on characterizing the effects of PLX4032 on five patient derived primary melanoma cell lines with respect to the MAPK signaling pathway and how modulation of this pathway by PLX4032 affects the migratory and invasive properties of the cells as well as the expression of various MAAs relevant to producing a multi-valent vaccine. Determination of the effects of PLX4032 on melanoma MAA expression will highlight its possible clinical use in combination treatments with immunotherapeutic vaccines and may lead to robust immune recognition and long-term anti-tumor responses in vivo.

\section{Materials and Methods}

\section{Cell Culture}

The primary allogeneic melanoma cell lines MEL-2, MEL-V, 3MM, KFM, and GLM-2 were isolat- 
ed from melanoma patients as previously described and informed consent was received from all subjects [4]. Primary melanoma cell lines were maintained in RPMI (Mediatech) supplemented with 10\% fetal bovine serum (FBS) (Atlanta Biologicals), $2 \mathrm{mM}$ L-glutamine (Mediatech), and Primocin ${ }^{\mathrm{TM}} 100 \mu \mathrm{g} / \mathrm{ml}$ (Invivogen). All primary lines were tested and determined to be free from mycoplasma as well as human and animal viruses (DNA Reference Lab Inc.). Established human melanoma cell lines SK-MEL-28 (ATCC \#HTB-72), SK-MEL-37 [14], and SK-MEL-103 [15] were generously gifted by Jedd $\mathrm{D}$. Wolchok, MD, PhD (Memorial Sloan-Kettering Cancer Center, New York, NY) and were cultured in RPMI supplemented with $10 \%$ FBS, $2 \mathrm{mM}$ L-glutamine, penicillin $10,000 \mathrm{IU} / \mathrm{ml}$, streptomycin 10,000ug/ml, and Normocin $^{\mathrm{TM}} 100 \mu \mathrm{g} / \mathrm{ml}$ (Invivogen).

\section{HLA Typing}

Low resolution HLA typing was done on six DNA samples using SSP UniTray HLA-ABC and HLA-DRDQ kits (Invitrogen Corporation, Brown Deer, WI), according to the manufacturer's procedure. DNA concentrations ranged from 92 to $112 \mathrm{ng} / \mathrm{mcL}$ with $260 \mathrm{~A} / 280 \mathrm{~A}$ ratios of 1.90 to 1.99. Amplicons were acquired on a QIAxcel System using a DNA Fast cartridge and QIAxcel ScreenGel software 1.0.0 (QIAGEN, Valencia, CA). Positive reactions were analyzed using UniMatch Plus 5.1 software (Invitrogen Corporation, Brown Deer, WI). HLA assignments are the generic/low resolution molecular HLA with the serological equivalent, when appropriate.

\section{BRAF Genotyping}

BRAF genotyping was carried out using the TaqMan real time PCR procedure as previously described [16] using an ABI Prism 7900HT (Applied Biosystems). Briefly, BRAF- (forward) 5'-CTACTGTT TTCCTTTACTTACTACACCTCAGA-3' and BRAF(reverse) 5'-ATCCAGACAACTGTTCAAACTGATG3' primers (900 nM; IDT) were used for amplification of the BRAF DNA. The wild-type probe 5'-VIC-CTAGCTACAGtGAAATC-3 and V600E mutant probe 5'-FAM-TAGCTACAGaGAAATC-3' (250 $\mathrm{nM})$ were used for allele discrimination. The genotyping reactions contained a final volume of $20 \mu \mathrm{l}$ and heated at $90^{\circ} \mathrm{C}$ for $10 \mathrm{~min}$, followed by 50 cycles $\left(92^{\circ} \mathrm{C}\right.$, $15 \mathrm{sec} ; 60^{\circ} \mathrm{C}, 1.5 \mathrm{~min}$ ) of amplification, followed by fluorescence detection.

\section{Western Blot Analysis}

Melanoma cells were harvested, washed with PBS, and lysed using the radioimmunoprecipitation assay (RIPA) buffer [50mM Tris- $\mathrm{HCl}(\mathrm{pH} 7.4), 150$ $\mathrm{mM} \mathrm{NaCl}, 0.2 \%$ sodium deoxycholate, $0.1 \% \mathrm{SDS}, 0.5 \%$
NP40] containing .1\% HALT protease/phosphatase inhibitor cocktail (Pierce). Protein concentration was determined using the Bio-Rad Protein Assay Dye based on the Bradford dye-binding method as previously described [4]. Cell lysates $(10 \mu \mathrm{g})$ were subjected to gradient $4-20 \%$ SDS-PAGE under reducing conditions. Proteins were transferred to Immobilon-P membranes (Millipore) at $220 \mathrm{~mA}$ for $2 \mathrm{hrs}$ and membranes were blocked in 5\% dried milk in TBST [200mM Tris- $\mathrm{HCl}, \mathrm{pH} 7.4,150 \mathrm{mM} \mathrm{NaCl}$, and $0.01 \%$ Tween-20 added fresh/liter of 1X TBS (TBS-T)]. Membranes were then incubated overnight at $4^{\circ} \mathrm{C}$ with either Gp100 (ABCAM), MART-1, MAGE-A1, Tyrosinase, Trp-1 (gp75) (Santa Cruz Biotechnology), BRAF, p-BRAF, MEK, p-MEK, ERK, p-ERK, GAPDH (Cell Signaling Technology), or Actin (Santacruz Biotechnology) primary antibodies. All primary antibodies were diluted at 1:200 in TBS-T containing 5\% milk. Membranes were subsequently washed with TBS-T and incubated with horseradish peroxidase (HRP) conjugated secondary antibodies (Pierce) in TBS-T containing $2 \%$ milk for $2 \mathrm{hrs}$ at room temperature while shaking. After washing, membranes were developed using ECL substrate (Pierce) and detected on HyBlot CL ${ }^{\mathrm{TM}}$ autoradiography film (Denville Scientific, Inc.).

\section{Transwell Migration and Invasion Assays}

To assess cellular migration in vitro, melanoma cells were harvested by trypsinization and $2.5 \times 10^{4}$ cells in $500 \mu \mathrm{l}$ of media were seeded in the upper chamber of BD Biocoat Control Inserts (BD Biosciences) with $8-\mu \mathrm{m}$ pore membrane filters. The lower chamber contained $750 \mu \mathrm{l}$ of media supplemented with 5\% FBS. After 18 hours, non-migratory cells were removed from the upper surface of the membrane using cotton tipped swabs. Migratory cells on the lower surface were then fixed using methanol and stained with $1 \%$ toluidine blue, $1 \%$ borax stain followed by two washes with distilled water. Inserts were then photographed and counted under (10X) magnification using bright field microscopy. Data are expressed as numbers of migrated cells per 10X field micrograph for each sample well and normalized to cell counts obtained from the untreated control.

To assess invasion in vitro, invasion assays were performed as above described using BD Biocoat Growth Factor Reduced Matrigel Invasion chambers (BD Biosciences) with $8-\mu \mathrm{m}$ pore membrane filters. Percent invasion (invasion index) was calculated based on the percent of cells invading through the growth factor reduced matrigel invasion chambers relative to the cells migrating through control membrane. 


\section{RNA Extraction/Analysis and qRT-PCR}

Total RNA was isolated using the Qiagen RNAeasy minikit (Qiagen) according to manufacturer's instructions RNA purity/concentration were evaluated as previously described [17]. The Applied Biosystems 7900HT Real Time PCR system (AppliedBiosystems) and SuperScript III Platinum SYBR ${ }^{\circledR}$ Green One-Step qRT-PCR system (Invitrogen) were used for qRT-PCR assays. Primers were purchased from Integrated DNA Technologies and designed using their PrimerQuest software (IDT). The reactions were performed using the following thermo cycling conditions: $50^{\circ} \mathrm{C}$ for $5 \mathrm{~min}, 95^{\circ} \mathrm{C}$ for $15 \mathrm{~min}$ and 40 cycles of $95^{\circ} \mathrm{C}$ for $15 \mathrm{~s}, 60^{\circ} \mathrm{C}$ for $30 \mathrm{~s}, 72^{\circ} \mathrm{C}$ for $30 \mathrm{~s}$. Samples were run in triplicate with the following primer sets: MART-1 (P1: 5'-CGG GAC AGC AAA GTG TCT CT-3', P2: 5'-AAG GTG GTG GTG ACT GTT CTG-3'), gp100 (P1: 5'-ACC AGA TAC TGA AGG GTG GCT-3', P2: 5'-CCA GGC ACA GGC ATG ATA AGC-3"', tyrosinase (P1: 5'-GAG GTC AGC ACC CCA CAA AT-3', P2: 5'-TGT TGT ACT CCT CCA ATC GGC-3'), MAGE-A1 (P1: 5'-GAG GTT TCC ATT CTG AGG GAC GG-3', P2: 5'-AAT CCT GTC CTC TGG GTT GGC-3' or the endogenous control glyceraldehydes-3-phosphate dehydrogenase (GAPDH) (P1: 5'-ACC ACA GTC CAT GCC ATC-3', P2: 5'-TCC ACC ACC CTG TTG CTG TA-3'). Data were analyzed according to the comparative $\mathrm{C}_{t}$ method and qRT-PCR product specificity was verified by melting curve analysis.

\section{Results}

Primary melanoma cell lines harbor mutations in the B-RAF signaling kinase. Mutations in the BRAF gene termed BRAF ${ }^{V 000}$ occur in over $60 \%$ of melanomas. We characterized the BRAF status of five primary, patient derived melanoma cell lines MEL-V, MEL-2, KFM, GLM-2, and 3MM. This analysis indicated that MEL-2 contained only the BRAFV600E allele, MEL-V, KFM, and GLM-2 contained both the wild type and BRAFV600E alleles, and 3MM contained only the wild type BRAF allele (Figure 1). As a control, we typed three established melanoma cell lines, namely SK-MEL-28, SK-MEL-37, and SK-MEL-103. In agreement with published data SK-MEL-28 contained only the BRAFV600E allele, SK-MEL-37 contained both the wild type and BRAFV600E alleles, and SK-MEL-103 contained only the wild type allele (Figure 1). Table 1 is a summary of the BRAFV600E mutational status for each cell line assayed. HLA typing was performed on each of the five primary cell lines. Results demonstrated a heterozygous expression of HLA haplotypes among all lines and thus can be used as a representative population (Table 2).

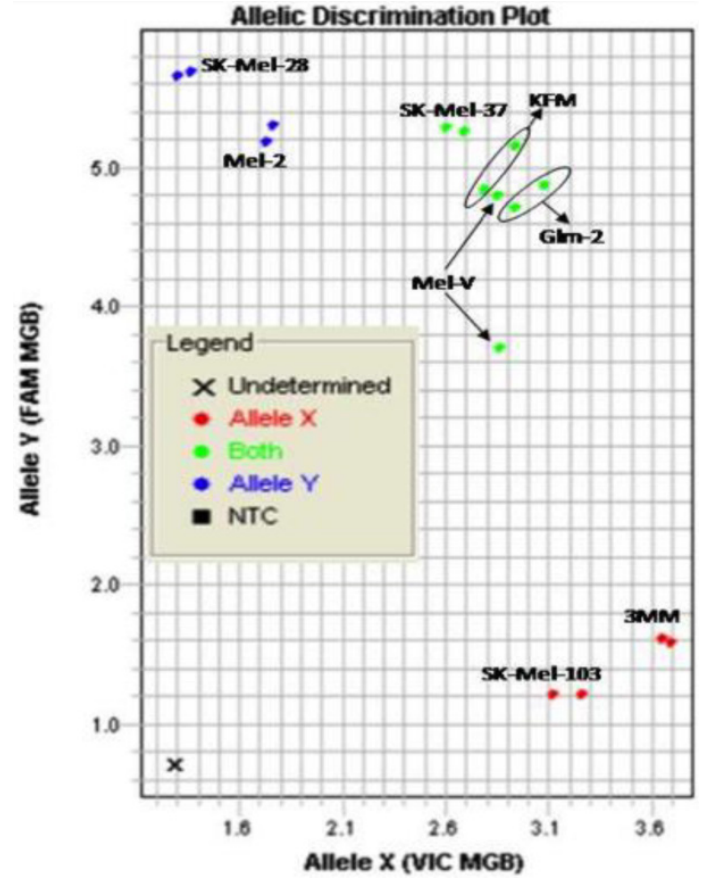

Figure 1. BRAF ${ }^{\mathrm{V} 600 \mathrm{E}}$ typing in melanoma. Five primary melanoma cell lines (MEL-2, MEL-V, KFM, GLM-2, and 3MM) and three established melanoma cell lines (SK-MEL-28, SK-MEL-37, SK-MEL-103) were typed for BRAFV600E status and allelic discrimination plot shown. Allele $Y$ refers to the BRAFV600E allele, Allele $X$ refers to the wild type BRAF allele. Typing was performed in duplicate samples, lines denoted in blue represent cells containing only mutant alleles, green denotes cell containing both mutant and wild type alleles, and red denoted cells only containing wild type alleles.

Table 1. BRAFV600E mutation status of typed lines.

\begin{tabular}{|l|l|}
\hline Melanoma Cell Line & BRAF $^{\text {6600E Status }}$ \\
\hline MEL-2 & Mutated \\
\hline MEL-V & Heterozygous \\
\hline $3 \mathrm{MM}$ & Wild Type \\
\hline KFM & Heterozygous \\
\hline GLM-2 & Heterozygous \\
\hline SK-MEL-28 & Mutated \\
\hline SK-MEL-37 & Heterozygous \\
\hline SK-MEL-103 & Wild Type \\
\hline
\end{tabular}

Table 2. Primary melanoma cell HLA typing.

\begin{tabular}{|c|c|}
\hline Melanoma Primary Cell line & HLA type \\
\hline Mel-v & $\begin{array}{l}A^{*} 01, A^{*} 26, B^{*} 35, B^{*} 44, C^{*} 04 \\
\text { DRB1*04, DRB1*07, DRB4*, DQB1*02, } \\
\text { DQB1*03(DQ8) }\end{array}$ \\
\hline Mel-2 & $\begin{array}{l}A^{*} 01, A^{*} 24, B^{*} 37, B^{*} 51, C^{*} 06, C^{*} 15 \\
\text { DRB1*01, DRB1*15, DRB5*, DQB1*05, DQB1*06 }\end{array}$ \\
\hline KFM & $\begin{array}{l}A^{*} 02, A^{*} 68, B^{*} 44, B^{*} 57, C^{*} 07, C^{*} 17 \\
\text { DRB1*07, DRB1*11, DRB3*, DQB1*03(DQ7), } \\
\text { DQB1*03(DQ9) }\end{array}$ \\
\hline $3 \mathrm{MM}$ & $\begin{array}{l}A^{*} 11, A^{*} 23, B^{*} 08, B^{*} 44, C^{*} 04, C^{*} 07 \\
\text { DRB1*03(DR17), DRB1*07, DRB3*, DRB4*, } \\
\text { DQB1*02 }\end{array}$ \\
\hline GLM-2 & $\begin{array}{l}A^{*} 02 \text { with rare } A^{*} 68, B^{*} 07, B^{*} 15(B 75), C^{*} 04, C^{*} 07 \\
\text { OR A*03 with rare } A^{*} 02, B^{*} 07, B^{*} 15(B 75), C^{*} 04, \\
C^{*} 07 \text { DRB1 } 08, \text { DRB1*16, DRB5*, DQB1*05, } \\
\text { DQB1*03(DQ7) }\end{array}$ \\
\hline
\end{tabular}


PLX4032 modulates MAPK signaling in melanoma cell lines. MAPK signaling is involved in many cellular processes including proliferation and migration. Cells harboring the BRAF ${ }^{\mathrm{V} 600 \mathrm{E}}$ mutation display a constitutively activated MAPK signaling pathway via increased phosphorylation of BRAF, MEK, and ERK. To determine the effect of PLX4032 on our primary melanoma cells, we assayed for the status of phosphorylated BRAF, MEK, and ERK via Western Blot analyses. Phosphorylation of BRAF, MEK, and ERK proteins was abrogated by PLX4032 treatment in both primary and established cells harboring the activating mutation (Figure 2A-D). Expression of unphosphorylated proteins was not altered by treatment. Treatment of the wild type cells 3MM and SK-MEL-103 led to an increased phosphorylation of the MAPK pathway proteins (Figure 2A,C-D), which is consistent with observations in literature [18]. Our results indicate that PLX4032 treatment of these primary cell lines leads to inhibition of MAPK signaling, however, determination of BRAF status in vivo is necessary as treatment activated signaling in wild type cells.
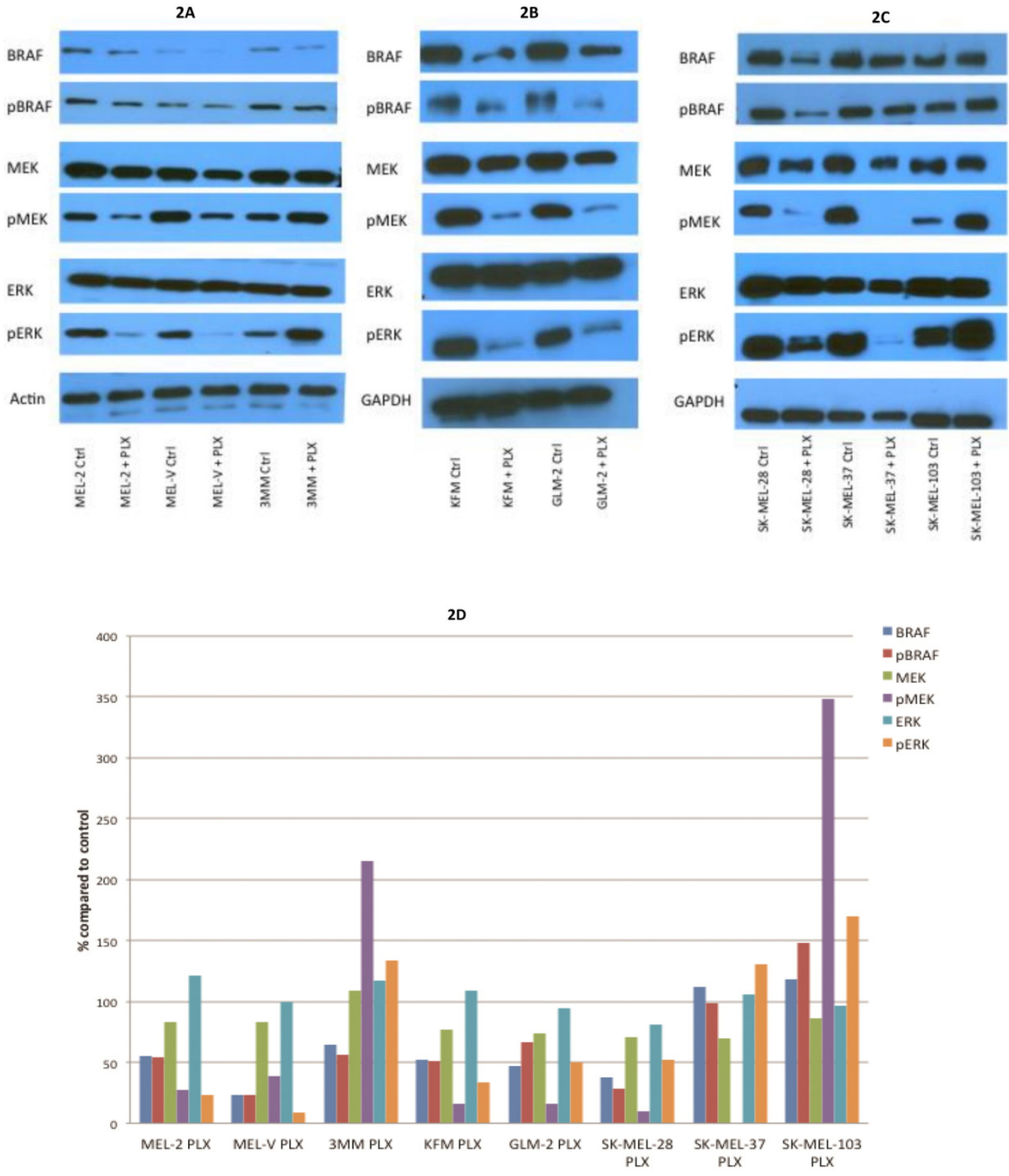

Figure 2. Effect of PLX4032 on MAPK signaling proteins in melanoma. Western blot analysis of phosphorylated BRAF, MEK, and ERK in primary melanoma lines MEL-2, MEL-V, and 3 MM (A), and KFM and GLM-2 (B) treated with $5 \mu$ M PLX4032 for 24 hours. Western blot analysis of phosphorylated BRAF, MEK, and ERK in established melanoma cell lines SK-MEL-28, SK-MEL-37, and SK-MEL-103 (C) treated with PLX4032 for 24 hrs. Actin and GAPDH served as a loading control. (D) Densitometric representation of Figures A-C expressed as a percentage of untreated control by Western blot analysis. 
PLX4032 modulates melanoma cell migration and invasion in vitro. Metastasis of melanoma is regulated by signals transmitted through the MAPK pathway. Previously we characterized the metastatic propensity of each of our primary lines taken from metastatic foci of melanoma patients $[4,19]$. To determine whether modulation of the MAPK pathway by PLX4032 could also abrogate melanoma cells' intrinsic invasiveness, we used a modified Boyden chamber assay to compare PLX4032 treatment of three primary cell lines representing the three BRAFV600E status groups, including MEL-2, GLM-2, and 3MM, and the three established cell lines to untreated controls. We observed only a moderate effect on cell migration (Figure $3,^{*} \mathrm{p}<.05$ ), however, PLX4032 abrogated cell invasion in the majority of BRAF ${ }^{\mathrm{V} 600 \mathrm{E}}$ positive lines (Figure 4). Most strikingly, in the cell line with the highest invasion index, GLM-2, PLX4032 treatment led to a significant $(* p<.05)$ nearly 20 fold reduction. Consistent with our Western blot observations, the invasive properties of the BRAF wild type $3 \mathrm{MM}$ and SK-Mel-103 cell lines were unaltered by PLX4032 treatment.

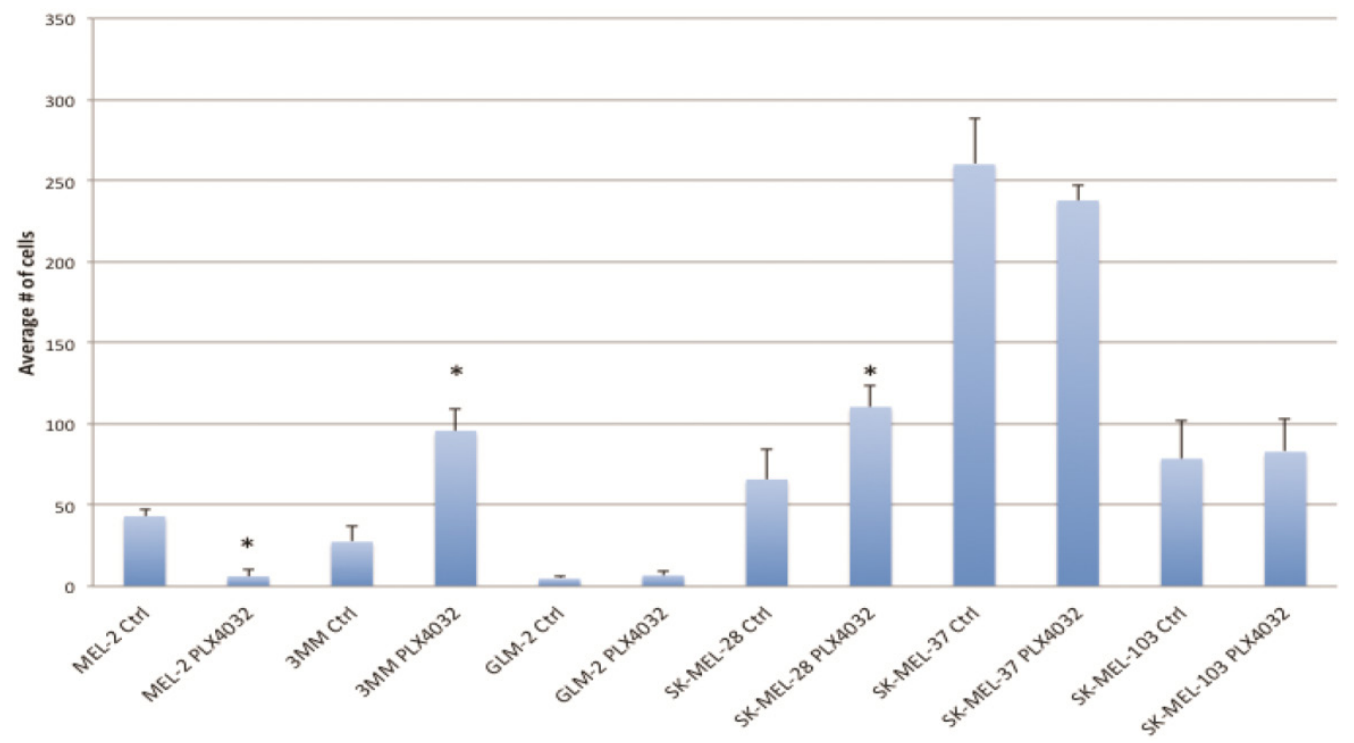

Figure 3. PLX4032 modulation of melanoma cell migration. Migration of human melanoma cells MEL-2, 3MM, GLM-2, SK-MEL-28, SK-MEL-37, and SK-MEL-103 \pm treatment of

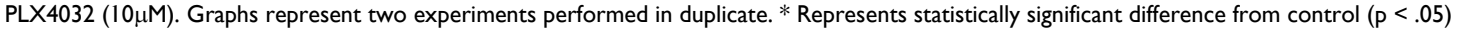

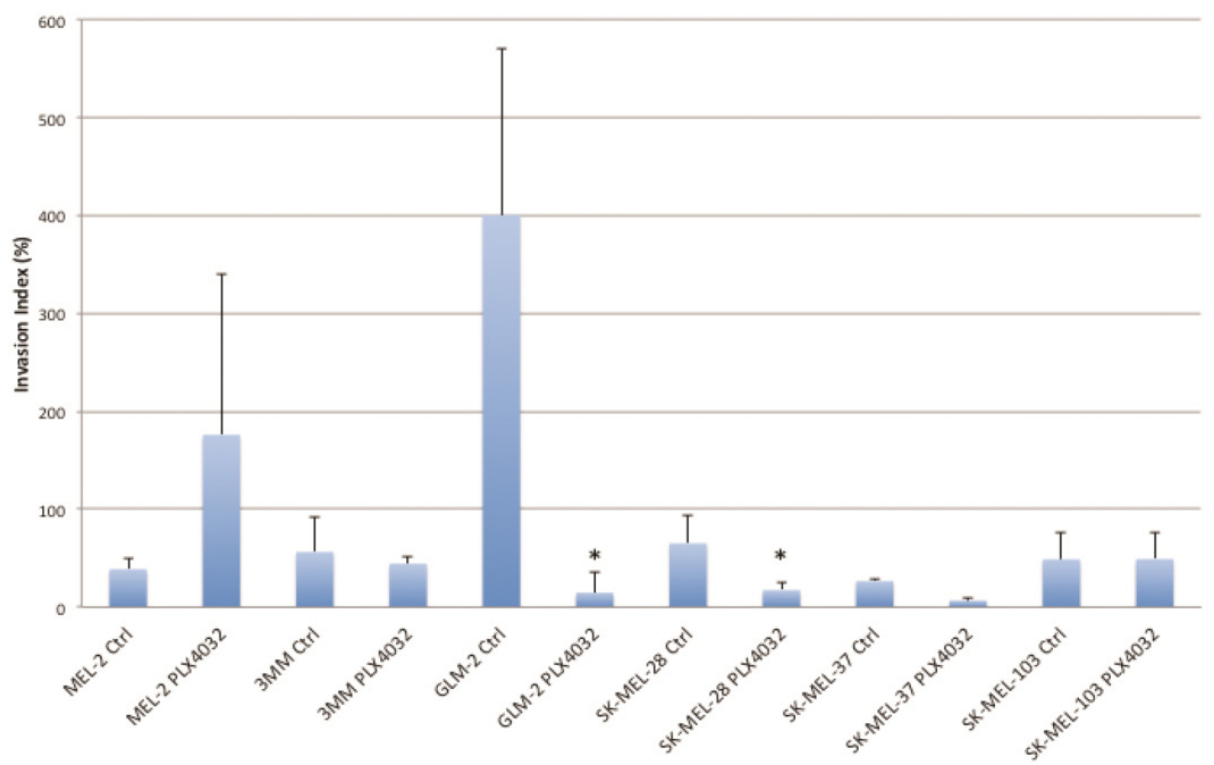

Figure 4. PLX4032 modulation of melanoma cell invasion. Invasion of human melanoma cells MEL-2, 3MM, GLM-2, SK-MEL-28, SK-MEL-37, and SK-MEL-103 with and without

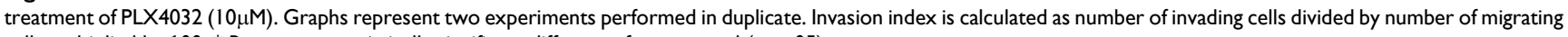
cells multiplied by 100. * Represents statistically significant difference from control $(p<.05)$ 
Inhibition of BRAFV600E increases MAA protein expression. Expression of various melanoma associated antigens (MAAs) supports the search for immunotherapeutic vaccines to treat melanoma. Recently, the MAPK pathway has been implicated in modulating the expression of various MAAs and thus may have implications in vaccine development. To determine whether PLX4032 treatment alters MAA expression in our primary cells we performed Western blot analysis. In this study, our observations indicate that the expression levels of the MAAs gp100, MART-1, tyrosinase, and Trp-1, all collectively involved in melanosomal maturation and melanin production, increased after treatment with PLX4032
(Figures 5 A, B). Strikingly, two of the primary lines, MEL-2 and KFM, expressed the MAA gp100 only after PLX4032 treatment. Interestingly, BRAF inhibition did not lead to an increase in all MAAs assayed as we observed a decrease in the cancer testis antigen MAGE-A1 protein levels in MAGE-A1+ cells after PLX4032 treatment. To determine whether PLX4032 modulates MAA expression transcriptionally, we performed RT-PCR analysis and observed a significant increase in mRNA levels of gp100, MART-1, and tyrosinase, as well as a decrease in MAGE-A1 in our primary lines (Figure 6), corresponding to results in protein expression.
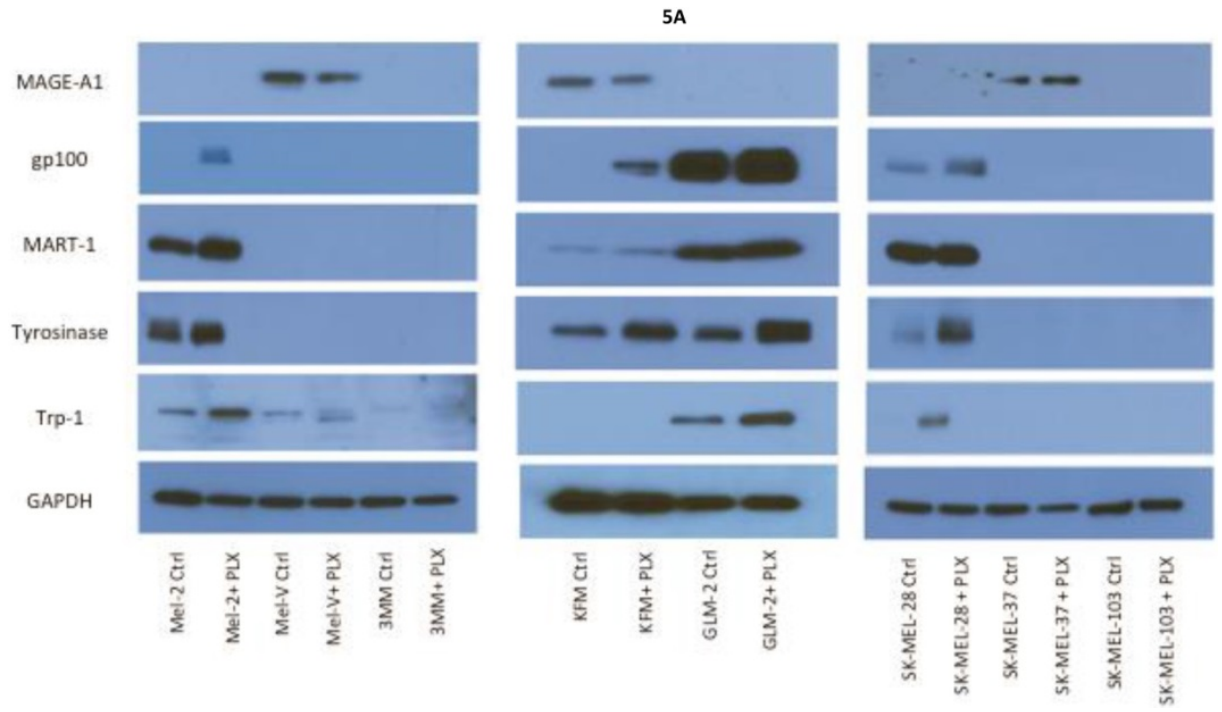

5B

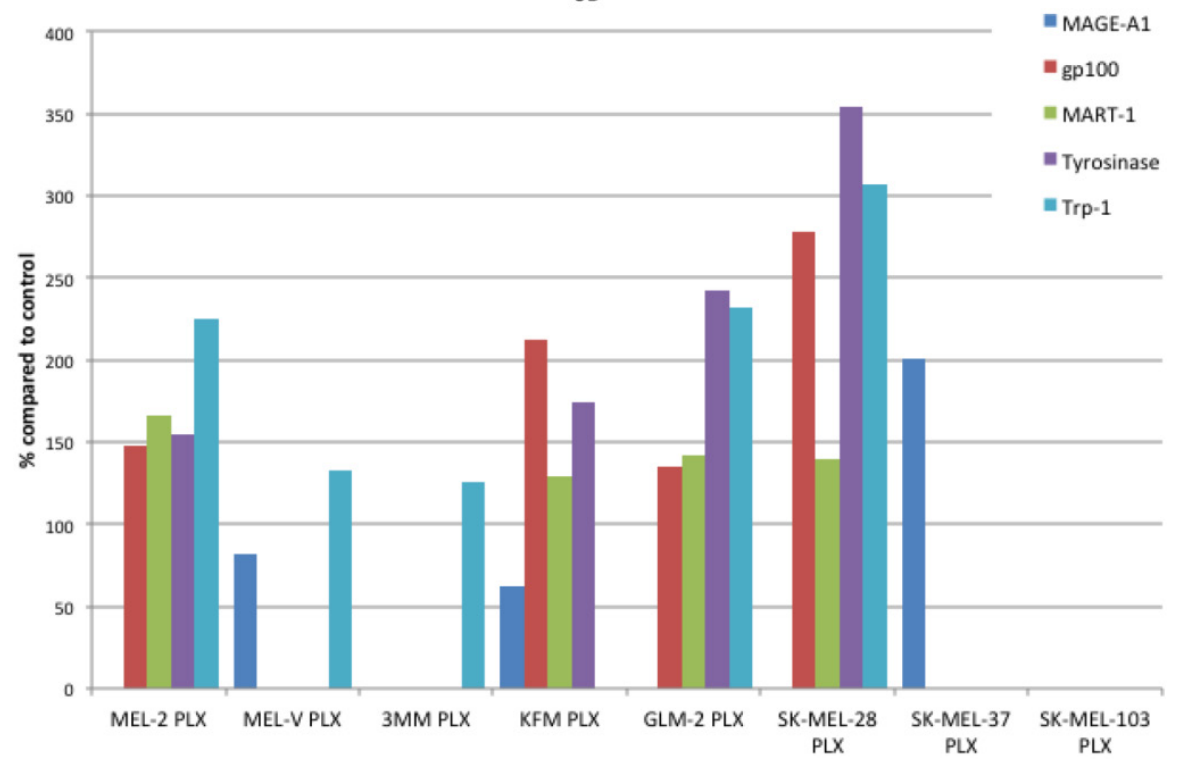

Figure 5. Melanoma associated antigen (MAA) expression up regulation by PLX4032. (A) Western blot analysis of MAA expression in primary and established melanoma cell lines with and without PLX4032 treatment (10 MM). (B) Densitometric representation of MAA expression expressed as a percentage of untreated control by Western blot analysis. 


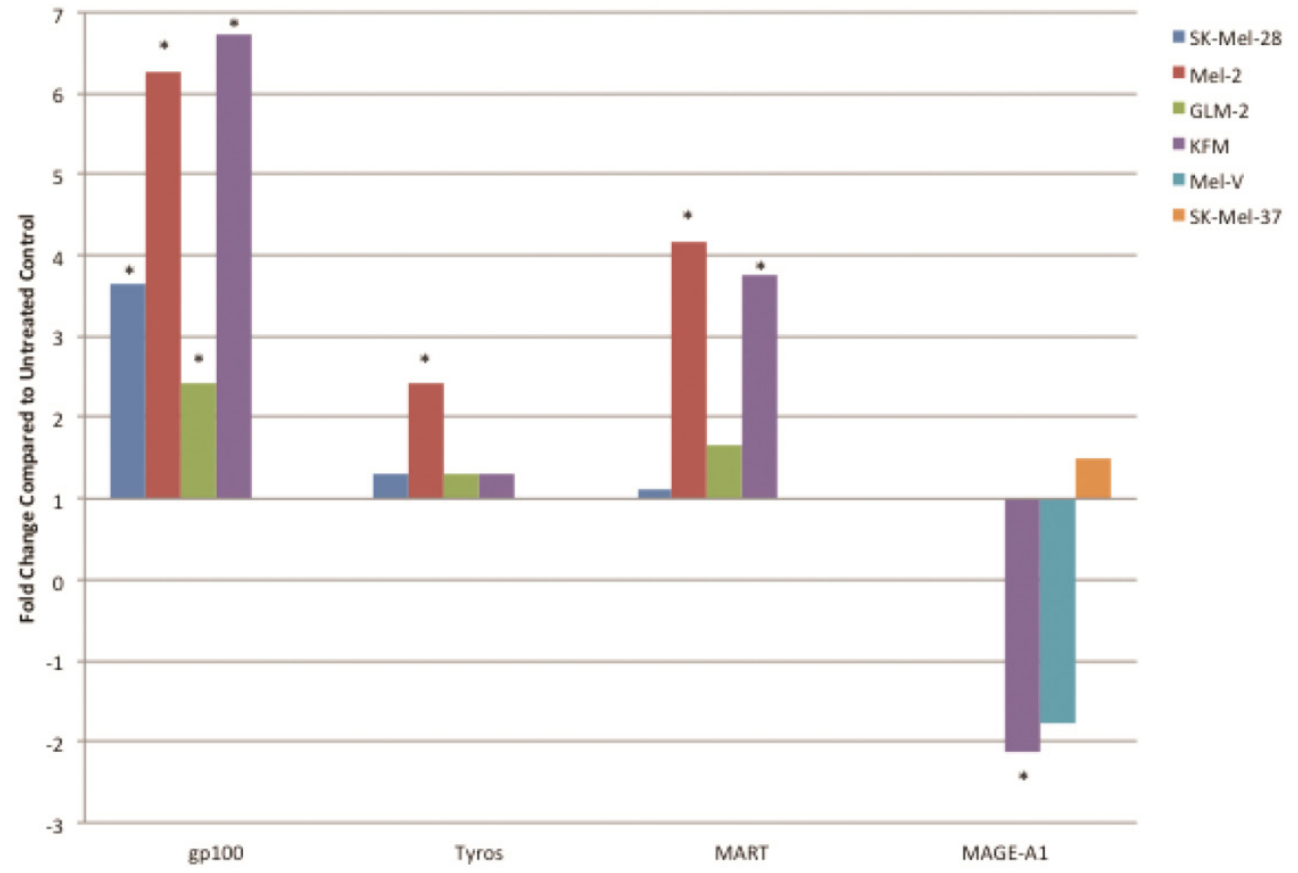

Figure 6. PLX4032 up regulates MAA RNA transcript levels. Increased levels of gp 100, tyrosinase, MART-1, and decrease levels of MAGE-A1 transcripts in PLX4032 treated $(10 \mu \mathrm{M})$ samples compared to untreated controls. Data represented as fold change compared to control. * Denotes significant fold change greater than 2.

\section{PLX4032 treatment increases MITF protein expression in primary melanoma cells}

The transcription factor MITF drives expression of MAAs and cell differentiation in melanoma, however loss of MAA expression during melanoma progression remains. To determine whether PLX4032 up regulation of MAA expression occurs via MITF up regulation we treated all primary and established cell lines with 5 and 10mM PLX4032 for 24 hours. Western blot analysis of MITF protein demonstrated an increase in 3 of the 4 primary BRAF mutated lines (Figure 7A, B). Consistent with literature the established cell lines (Sk-Mel-28, 37, and 103) did not express MITF [20]. Further, the non-mutated primary cell line $3 \mathrm{MM}$ also did not express detectable levels of MITF protein. Only one cell line, GLM-2, had reduced MITF expression after PLX4032 treatment. Our results demonstrate that inactivation of MAPK signaling via BRAF inhibition up regulates expression of multiple melanoma associated antigens likely through increased expression of MITF protein (Figure 8).

\section{Discussion}

Under normal physiological conditions, cells require defined intracellular signaling events relayed through signaling pathways to ensure controlled cell turnover. The MAPK pathway consists of a cascade of kinases including the GTPase Ras, followed by BRAF, MEK, and ERK, leading to activation of various tran- scription factors involved in normal cell growth and regeneration [21, 22]. Such processes include cell cycle progression and cell proliferation as well as cell migration and invasion [23-27]. Mutations arising in these signaling molecules generate de-regulation of cell phenotype characterized by high rates of proliferation and generation of cell types into increased migration and invasion.

Deregulation of the MAPK pathway is frequently observed in melanoma in which nearly a quarter contain mutations in NRAS and greater than half possess an activating mutation in the BRAF protein kinase known as BRAFV600E $[11,28]$. Mutation of BRAF leads to constitutive activation of ERK, which has been shown to increase melanoma proliferation and migration through increased expression of $\alpha_{V} \beta_{3}$-integrin and IL-8 [29]. In our study, we observed a similar $\mathrm{BRAF}^{\mathrm{V} 600 \mathrm{E}+}$ mutation rate as four of the five primary melanoma lines used contained the BRAFV600E allele. PLX4032 functions by targeting the BRAFV600E protein preventing downstream phosphorylation of MEK and ERK. Currently, PLX4032 is in use in melanoma clinical trials and has led to promising results in patients harboring the mutation however significant development of drug resistance has also been noted. Interestingly, a secondary effect of PLX4032 treatment was observed by Boni et al that demonstrated inhibition of BRAFV600E increased activity of microphthalmia transcription factor (MITF). 
7A
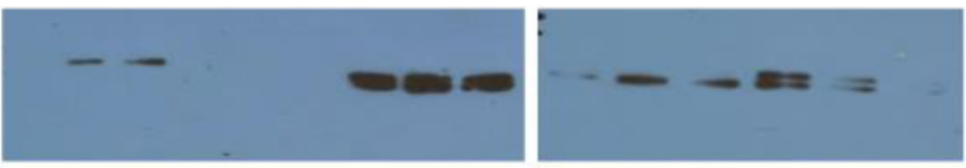

GAPDH
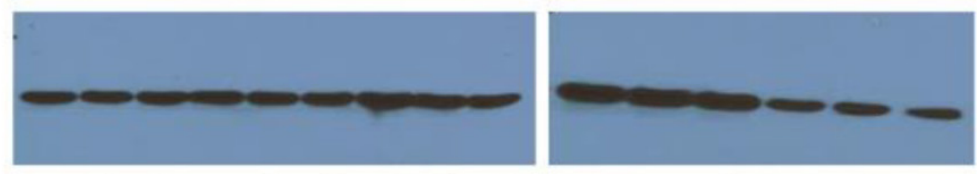

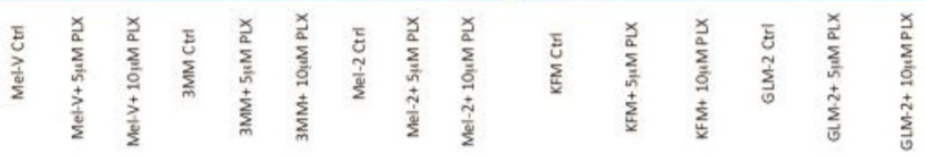

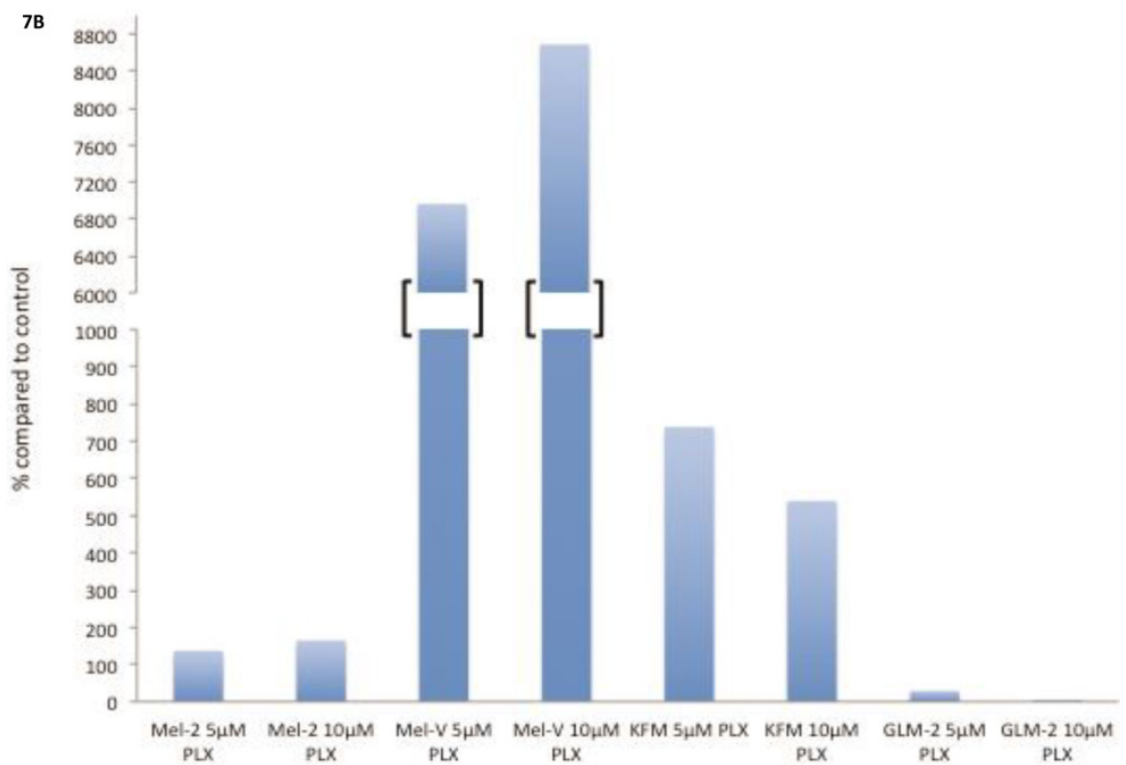

Figure 7. BRAF inhibition leads to an up regulation of MITF in primary melanoma cell lines. (A) Western blot analysis of MITF protein in primary cell lines Mel-V, Mel-2, KFM, and GLM-2 treated with $5 \mu \mathrm{M}$ and 10 $\mathrm{M}$ PLX4032 for 24 hours. (B) Densitometric representation of Figure A expressed as percent change from untreated control by Western Blot analysis.

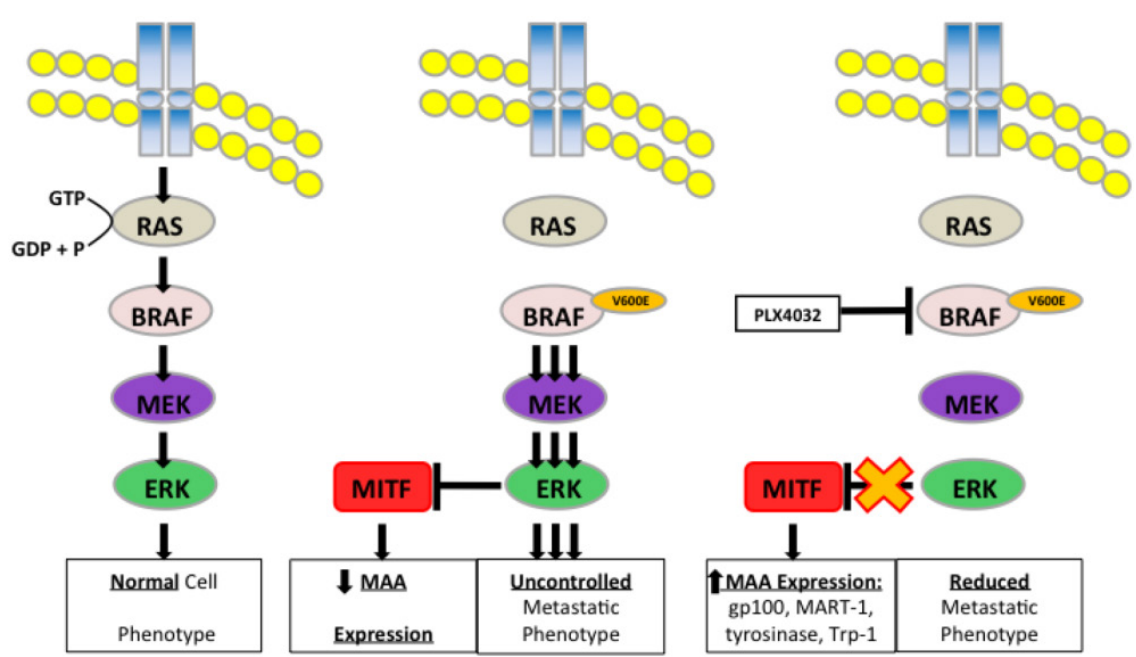

Figure 8. Schematic representation of PLX4032 modulation of the MAPK pathway and MAA expression.

MITF is a tissue specific transcription factor involved in melanosome differentiation and melanin production. MITF regulated genes include MAAs such as tyrosinase, Trp-1, MART-1, and gp100 [30-32]. Interestingly previous work has demonstrated that during melanoma progression as cells acquire a metastatic phenotype MITF levels diminish and abrogation of MITF activity was due to increased MAPK signaling [7, 33]. Melanoma cell treatment with PLX4032 blocked constitutive MAPK signaling via 
BRAFV600E and led to restoration of MITF activity and expression of the MAA gp100. Thus a close relationship exists between the MAPK pathway and the expression of MAAs, and as such BRAFV600E is in part inversely responsible for the immunogenicity associated with melanoma and has clinical implications for use in immunotherapy.

Previously, we designed a vaccinia melanoma oncolysate $(\mathrm{VMO})$ vaccine from five primary ex vivo derived melanoma cell lines and conducted a Phase III clinical trial to monitor disease-free interval and overall survival length in patients from 11 institutions [4]. VMO treatment led to a $10 \%$ increase in survival advantage particularly in the subset group of males aged 44-57 years, demonstrating that our vaccine based immunotherapy is relevant and may be used to treat melanoma patients. Recent advances in melanoma treatments using CTLA-4 and PD- 1 as targets have yielded significant clinical successes [34, 35]. The release of checkpoint inhibitors demonstrates the viability of immunotherapeutic approaches in melanoma. We believe that can be achieved by a combination of positive stimulators such as our multi-valent vaccine and negative checkpoint inhibitors and is supported by recent clinical trials investigating MAA expression in patients treated with BRAF inhibitors that elicits an enhanced T-cell response as well as an influx of monocyte derived dendritic cells [36, 37]. Characterization of the primary cells used to generate the VMO based on known MAA expression, and thus proteins important for immune recognition during vaccine administration, revealed differential expression among each of the cell lines of various MAAs including gp100, tyrosinase, and MART-1 as previously shown. Importantly, not all cell lines expressed all of the MAAs investigated, providing further support for the use of our multi-valent vaccine therapy. These findings, together with observed frequencies of BRAF mutations and the presence and absence of the BRAF ${ }^{V 600 E}$ mutation in the different primary cell lines provided us with a controlled experimental model to investigate the relationship between MAA expression and PLX4032 activity.

This study demonstrates that PLX4032 abrogates MAPK signaling (specifically phosphorylation of $\mathrm{BRAF}, \mathrm{MEK}$, and ERK) in BRAFV600E+ primary melanoma cells and that treatment also diminishes tumor cell metastatic phenotype in vitro. Further PLX4032 treatment increased expression of MAAs including tyrosinase, MART-1, and strikingly gp100, which only became detectable in two of the primary lines after treatment. Increases in MAA expression was due to increased transcription of MAA genes and correlated with changes in protein levels observed. Interestingly, not all MAAs increased upon treatment, as we ob- served a decrease in MAGE-A1 levels in KFM and MEL-V. Importantly to note, PLX4032 treatment of both primary and established cells expressing normal BRAF protein led to activation of MAPK signaling. These findings have a vast impact for the role of PLX4032 as a combination strategy with immunotherapeutic vaccine administration in $\mathrm{BRAF}^{\mathrm{V} 600 \mathrm{E}+}$ melanoma patients. This strategy is supported further by observations that PLX4032, while targeting mutant melanoma, does not alter T-cell function and enhances tumor recognition [13].

Taken together, overwhelming evidence of the early effectiveness of PLX4032 treatment in melanoma together with our demonstration that PLX4032 treatment also leads to increased MAA expression, provides a strong case for its use in combination with our immunotherapeutic vaccinia melanoma oncolysate vaccine which may lead to a robust immune response and prolonged tumor regression in melanoma patients. Through this combinational strategy, PLX4032 treatment will aid in initial anti-tumor activities, including reduced tumor proliferation, as well as the important secondary effect of enhanced MAA expression, which may aid in T-cell-tumor recognition during vaccine administration. This early detection by the immune response is crucial and may prevent generation of drug resistance and tumor recurrence in melanoma patients. However, while a robust stimulation of the immune response is important for vaccine effectiveness, it is essential to further block any negative down regulation of immune cell function through immune checkpoint blockade. Currently, ipilimumab, a fully human monoclonal antibody that targets cytotoxic T-lymphocyte associated antigen 4 (CTLA-4) as well as antibodies targeting programmed cell death 1 (PD-1) proteins have demonstrated some effectiveness in melanoma patients through prolongation of active T-cell responses [38-40]. The effectiveness of immune checkpoint blockade in combination with our vaccine-PLX4032 model requires further study.

\section{Abbreviations}

MAA: melanoma associated antigens; TRP-1: tyrosinase related protein-1; gp100: glycoprotein 100; MART-1: melanoma antigen recognized by $\mathrm{T}$ cells 1 ; MAGE-A1: melanoma-associated antigen 1; FDA: Food and Drug Administration; NCI: National Cancer Institute; IL-2: interleukin-2; MITF: microphthalmia-associated transcription factor; MAPK: mitogen activated protein kinase; IL-10: interleukin-10; VEGF: vascular endothelial growth factor; IL-6: interleukin-6; BRAF: v-raf murine sarcoma viral oncogene homolog B; MEK: mitogen-activated protein/extracellular signal-regulated kinase kinase; ERK: extracellu- 
lar-signal-regulated kinases; FBS: fetal bovine serum; PCR: polymerase chain reaction; DNA: deoxyribonucleic acid; RIPA: radioimmunoprecipitation assay; TRIS-HCL: tris(hydroxymethyl)aminomethanehydrogen chloride; $\mathrm{NaCl}$ : sodium chloride; SDS: sodium dodecyl sulfate; TBS-T: tris buffered saline-tween 20; GAPDH: glyceraldehyde 3-phosphate dehydrogenase; HRP: horseradish peroxidase; qRT-PCR: quantitative real-time polymerase chain reaction; Ras: rat sarcoma; VMO: vaccinia melanoma oncolysate; CTLA-4: cytotoxic T-lymphocyte associated antigen 4; PD-1: programmed cell death 1 .

\section{Acknowledgements}

This research project was funded by NCI grant (RO1CA4538-01A1).

\section{Competing Interests}

The authors have declared that no competing interest exists.

\section{References}

1. Dunn GP, Lewis JS, Sunwoo JB, et al. Spontaneous regression of cutaneous head and neck melanoma: implications for the immunologic control of neoplasia. Head Neck. 2008; 30: 267-272.

2. Printz C. Spontaneous regression of melanoma may offer insight into cancer immunology. J Natl Cancer Inst. 2001; 93: 1047-1048.

3. Gattoni-Celli S, Cole DJ. Melanoma-associated tumor antigens and their clinical relevance to immunotherapy. Semin Oncol. 1996; 23: 754-758.

4. Suriano R, Rajoria S, George AL, et al. Ex vivo derived primary melanoma cells: Implications for immunotherapeutic vaccines. J of Cancer. 2013; 4: 371-382.

5. Schwartzentruber DJ, Lawson DH, Richards JM, et al. gp100 peptide vaccine and interleukin-2 in patients with advanced melanoma. N Engl J Med. 2011; 364: 2119-2127.

6. Escudier B, Dorval T, Chaput N, et al. Vaccination of metastatic melanoma patients with autologous dendritic cell (DC) derived-exosomes: results of the first phase I clinical trial. J Transl Med. 2005; 3: 10-23.

7. Levy C, Khaled M, Fisher DE. MITF: master regulator of melanocyte development and melanoma oncogene. Trends Mol Med. 2006; 12: 406-414.

8. Wu M, Hemesath TJ, Takemoto CM, et al. c-Kit triggers dual phosphorylations, which couple activation and degradation of the essential melanocyte factor Mi. Genes Dev. 2000; 14: 301-312.

9. Dhillon AS, Hagan S, Rath O, et al. MAP kinase signaling pathways in cancer. Oncogene. 2007; 26: 3279-3290.

10. Sumimoto $H$, Imabayashi $F$, Iwata $T$, et al. The BRAF-MAPK signaling pathway is essential for cancer-immune evasion in human melanoma cells. JEM. 2006; 203: 1651-1656.

11. Davies H, Bignell GR, Cox C, et al. Mutations of the BRAF gene in human cancer. Nature. 2002; 417: 949-954.

12. Chapman $\mathrm{PB}$, Hauschild $\mathrm{A}$, Robert $\mathrm{C}$, et al. Improved survival with vemurafenib in melanoma with BRAF V600E mutation. N Engl J Med. 2011; 364: 2507-2516.

13. Boni A, Cogdill AP, Dang P, et al. Selective BRAFV600E inhibition enhances T-cell recognition of melanoma without affecting lymphocyte function. Cancer Res. 2010; 70: 5213-5219.

14. Winchester RJ, Wang CY, Gibofsky A, Kunkel HG, Lloyd KO, Old LJ. Expression of la-like antigens on cultured human malignant melanoma cell lines. Proc Natl Acad Sci. 1978; 75: 6235-6239.

15. Kaufmann WK, Nevis KR, Qu P, et al. Defective cell cycle checkpoint functions in melanoma are associated with altered patterns of gene expression. J Invest Dermatol. 2008; 128: 175-187.

16. Benlloch S, Paya A, Alenda C, et al. Detection of BRAF V600E mutation in colorectal cancer; comparison of automatic sequencing and real-time chemistry methodology. J Mol Diagn. 2006; 8: 540-543.

17. Nowicki TS, Kummer NT, Iacob C, et al. Inhibition of uPAR and UPA reduces invasion in papillary thyroid carcinoma cells. Laryngoscope. 2010; 120: 1383-1390.

18. Halaban R, Zhang W, Bacchiocchi A, et al. PLX4032, a selective BRAFV600E kinase inhibitor, activates the ERK pathway and enhances cell migration and proliferation of BRAFWT melanoma cells. Pigment Cell Melanoma Res. 2010; 23: $190-200$
19. Suriano R, Rajoria S, George AL, et al. Follow-up analysis of a randomized phase III immunotherapeutic clinical trial on melanoma. Mol and Clin Oncology. 2013; 1: 466-472.

20. Hoek KS, Schlegel NC, Eichhoff OM, et al. Novel MITF targets identified using a two-step DNA microarray strategy. Pigment Cell Melanoma Res. 2008; 21: 665-676.

21. Karin M. The regulation of AP-1 activity by mitogen-activated protein kinases. J Biol Chem. 1995; 270: 16483-16486.

22. Dhawan P, Richmond A. A novel NF-kappa B-inducing kinase-MAPK signaling pathway up-regulates NF-kappa B activity in melanoma cells. J Biol Chem. 2002; 277: 7920-7928.

23. Lavoie JN, L Allemain G, Brunet A, et al. Cyclin D1 expression is regulated positively by the $\mathrm{p} 42 / \mathrm{p} 44^{\mathrm{MPAK}}$ and regulated by the p38/HGGMAPK pathway. J Biol Chem. 1996; 271: 20608-20616.

24. Balmanno K, Cook SJ. Sustained MAP kinase activation is required for the expression of cyclin D1, p21Cip1 and a subset of AP-1 proteins in CCL39 cells. Oncogene. 1999; 18: 3085-3097.

25. Blanchard DA, Mouharmad S, Auffredou M-T, et al. Cdk2 associates with MAP kinase in vivo and its nuclear translocation is dependent on MAP kinase activation in IL-2 dependent kit 225 T lymphocytes. Oncogene. 2000; 19: 4184-4189.

26. Choudhury GG, Karamitsos C, Hernandez J, et al. PI-3-kinase and MAPK regulate mesangial cell proliferation and migration response to PDGF. AJP-Renal Physiol. 1997; 273: 931-938.

27. Huang C, Jacobson K, Schaller MD. MAP kinases and cell migration. J Cell Sci. 2004; 117: 4619-4628

28. Sekulic A, Haluska P Jr, Miller AJ, et al. Malignant melanoma in the $21^{\text {st }}$ century; the emerging molecular landscape. Mayo Clin Proc. 2008; 83: 825-846.

29. Estrada Y, Dong J, Ossowski L. Positive crosstalk between ERK and p38 in melanoma stimulates migration and in vivo proliferation. Pigment Cell Melanoma Res. 2009; 22: 66-76.

30. Bentley NJ, Eisen T, Goding CR. Melanocyte-specific expression of the human tyrosinase promoter: activation by the microphthalmia gene product and role of the initiator. Mol Cell Biol. 1994; 14: 7996-8006.

31. Yasumoto $\mathrm{K}$, Mahalingam $\mathrm{H}$, Suzuki $\mathrm{H}$, et al. Transcriptional activation of the melanocyte-specific genes by the human homolog of the mouse Microphthalmia protein. J Biochem. 1995; 118: 874-881.

32. Du J, Miller AJ, Widlund HR, et al. MLANA/MART1 and SILV/PMEL17/GP100 are transcriptionally regulated by MITF in melanocytes and melanoma. Am J Pathol. 2003; 163: 333-343.

33. Salti GI, Manougian T, Farolan M, et al. Micropthalmia transcription factor: a new prognostic marker in intermediate-thickness cutaneous malignant melanoma. Cancer Res. 2000; 60: 5012-6.

34. Barbee MS, Ogunniyi A, Horvat TZ, et al. Current status and future directions of the immune checkpoint inhibitors ipilimumab, pembrolizumab, and nivolumab in oncology. Ann Pharmacother. 2015; [Epub ahead of print].

35. Mahoney KM, Freeman GJ, McDermott DF. The next immune-checkpoint inhibitors: PD-1/PD-L1 blockade in melanoma. Clin Ther. 2015; 37(4): 764-782.

36. Frederick DT, Piris A, Cogdill AP, et al. BRAF inhibition is associated with enhanced melanoma antigen expression and a more favorable tumor microenvironment in patients with metastatic melanoma. Clin Cancer Res. 2013; 19(5): 1225-1231.

37. Vella LJ, Pasam A, Dimopoulos N, et al. MEK inhibition, alone or in combination with BRAF inhibition, affects multiple functions of isolated normal human lymphocytes and dendritic cells. Cancer Immunol Res. 2014; 2(4): 351-360.

38. Wolchok JD, Saenger Y. The mechanism of anti-CTLA-4 activity and the negative regulation of T-cell activation. Oncologist. 2008; 13: 2-9.

39. Prieto PA, Yang JC, Sherry RM, et al. CTLA-4 blockade with ipilimumab: long-term follow-up of 177 patients with metastatic melanoma. Clin Cancer Res. 2012; 18: 2039-2047.

40. Topalian SL, Hodi FS, Brahmer JR, et al. Safety, activity, and immune correlates of anti-PD-1 antibody in cancer. N Engl J Med. 2012; 366: 2443-2454. 